Article

\title{
From Erosion to Remineralization: The Possible Role of Two Topic Home Devices Used as Combined Treatment
}

\author{
Lidia Fanfoni ${ }^{1}$, Fulvia Costantinides ${ }^{1}$, Federico Berton ${ }^{1} * *$ () Giulio Marchesi ${ }^{1}$, Leila Polo ${ }^{2}$, \\ Roberto Di Lenarda ${ }^{1}$ and Vanessa Nicolin ${ }^{3}$ \\ 1 Clinical Department of Medical, Surgical and Health Sciences, University of Trieste, 34127 Trieste, Italy; \\ lidia.fanfoni@libero.it (L.F.); F.COSTANTINIDES@fmc.units.it (F.C.); gmarchesi@units.it (G.M.); \\ rdilenarda@units.it (R.D.L.) \\ 2 School of Dental Sciences, University of Trieste, 34127 Trieste, Italy; s233072@ds.units.it \\ 3 Bone Lab, Clinical Department of Medical, Surgical and Health Sciences, University of Trieste, 34127 Trieste, \\ Italy; nicolin@units.it \\ * Correspondence: fberton@units.it; Tel.: +39-040-399-2020
}

Received: 11 May 2020; Accepted: 12 June 2020; Published: 14 June 2020

\begin{abstract}
Severe dental erosion could be one of the complications of gastroesophageal reflux disease and food disorders such as bulimia nervosa. The aim of the present in vitro study was to evaluate the remineralization efficiency and the erosion prevention capability obtained by combining the use of Elmex Erosion Protection (Elm-EP) and GC Tooth Mousse (GC-TM) in cases of strongly eroded enamel surfaces. Twenty-four specimens of bovine tooth were superficially treated with hydrochloric acid to mimic severe erosion conditions. The specimens were divided into 3 groups accordingly to a different remineralizing treatment for 15 days: immersion in artificial remineralizing saliva, brushing with Elm-EP and brushing with Elm-EP in association with a daily application of GC-TM paste. After the remineralization procedures, the specimens were exposed to a treatment with acid. The effectiveness of the treatments were assessed by surface profilometric analysis and scanning electron microscopy at four different steps. Furthermore, the quantity of the $\mathrm{Ca}^{2+}$ lost during erosion was determined. The combined action of Elm-EP and GC-TM led to a 50\% roughness decrease of critically eroded dental surfaces. The tandem use of Elmex Erosion Protection and GC Tooth Mousse resulted a promising protecting strategy for the prevention of the dental mineral loss.
\end{abstract}

Keywords: erosion protection; severe dental erosion; remineralizing agents; profilometric analysis; scanning electron microscopy

\section{Introduction}

Dental erosion is defined as a chemical (acid-related) loss of dental hard tissue with a process independent from bacteria [1,2]. Concerning the origin of the erosion-causing acid, a distinction between exogenous and endogenous erosion could be made [3]: extrinsic factors include the dietary regimen and the use of some medications, while intrinsic factors are mostly correlated to diseases such as gastroesophageal reflux (GERD), often affecting diabetic and obese individuals, bulimia nervosa (BN) and alcoholism [2]. The initial stage of dental erosion, that is a reversible process, is characterized by the softening of the enamel due to acidic demineralization of hydroxyapatite (HA) [4]. For this reason, the partially demineralized HA crystals may re-establish the original conformation if exposed to a favorable oral environment, at this stage [2]. The intrinsic remineralization due to the chemical composition of saliva is well documented, in fact at physiological $\mathrm{pH}$, saliva is supersaturated with phosphoprotein-stabilized $\mathrm{Ca}^{2+}$ and $\mathrm{PO}_{4}{ }^{3-}$ ions, which are bioavailable for hard tissue development 
and maintenance, establishing an homeostatic equilibrium. However, salivary remineralization is a slow process, affecting only the surface of mild eroded tooth and improving neither the aesthetics nor the structural properties, in case of subsurface lesions [5]. Indeed, in case of patients affected by chronic GERD and BN, the self salivary remineralization process results inadequate: as the acidic impact persists, additional dissolution of the enamel matrix occurs, resulting in the layer-by-layer inorganic material loss, decrease of the enamel thickness and, occasionally, in dentin exposure [4]. This chronic situation, often leading to a painful experience for patients, requires professional dental assistance and, at the same time, the use of specific remineralizing and wear-protecting agents [6]. Fluorinated toothpastes are the most commonly used remineralizing agents that convert hydroxyapatite (HA) into the stronger fluoro-hydroxyapatite, which is more resistant to the acid wear [7].

Despite the use of remineralizing agents able to limit the inconveniences of drastic dental erosion, it is considered necessary the development of new combined strategies with the aim to avert hard tissue loss. To exert an antierosive effect, conventional fluorides (such as sodium fluoride (NaF)) appear to require an intensive and unrealistic fluoridation regimen [4]. On the other hand, the increase in prevalence of dental erosion among population, despite the use of fluoride-containing toothpastes, points out the need for more effective compounds [5]. Stannous fluoride $\left(\mathrm{SnF}_{2}\right)$, titanium tetrafluoride, amine fluoride, $\mathrm{HA}$ nanoparticles, $\mathrm{SnF}_{2}$ with amorphous calcium phosphate, zinc-carbonate-HA nanoparticles, potassium nitrate, chitosan, and various proteins, are only some examples of the developed components of toothpastes which efficacy in preventing enamel and dentin erosion have been investigated in the last years [8-11]. Under these considerations, the combination between the remineralizing capacity and the promotion of surface resistance become an advisable goal for contemporary commercial products, towards an efficient treatment of patients afflicted by chronic dental erosions.

At the state of the art, several studies showed the performances of different suitable agents with anti-erosive properties or remineralizing activity [12], but to the authors' knowledge, there is a lack of information regarding the combined use of these products. The present study aims to investigate the remineralization capability and the tooth-surface protecting attitude achievable combining two commonly used home-devices in case of teeth characterized by severe enamel erosion conditions. The toothpaste Elmex Erosion Protection (Elm-EP, Colgate-Palmolive Co., New York, USA), based on the ChitoActive complex (Chitosan/AmF/SnCl 2 ) and the GC Tooth Mousse (GC-TM, GC Co., Tokyo, Japan) containing milk-derived casein-phospholipid and amorphous calcium phosphate (CPP-ACP), were specifically chosen because of their differences both in composition and mode of action: Elm-EP is a toothpaste based on chitosan $(0.5 \%)$ and enriched with amine fluoride, sodium fluoride (1400 ppm total $\mathrm{F}^{-}$) and $\mathrm{SnCl}_{2}$ (3500 ppm) [11]. Biopolymer chitosan, derived from chemical deacetylation of chitin, has been largely studied for use in preventive dentistry. Its anti-inflammatory, antimicrobial and biofilm inhibition properties have been confirmed as its ability to adhere to hydroxyapatite and to prevent dental wear [13-15]. Nevertheless, chitosan does not contribute to the remineralization of existing dental lesions [16]. On the other hand, chitosan microparticles act as carriers for the delivery of $\mathrm{F}^{-}$in the oral cavity [17]. Fluoride ions, derived from $\mathrm{NaF}$ and $\mathrm{AmF}$, displace hydroxyapatite hydroxide groups forming fluoro-hydroxyapatite playing a double role in the surface-protection process: first, fluoride acts as a catalyst, assisting in the remineralization of enamel with phosphate ions dissolved in saliva; secondly the decreased solubility of fluoro-hydroxyapatite crystals ensures a superior resistance to acid erosion [2]. Finally, the $\mathrm{Sn}^{2+}$ ions interacting with hydroxyapatite may lead to the precipitation of a protective layer of insoluble mixed tin-salts which inhibits mineral loss protecting the tooth surface [18].

Differently, CG-TM has been indicated as fluoride-free remineralizing agent, containing milk-derived multiphosphorylated casein phosphopeptides (CPP) able to form stable complexes with calcium and phosphate ions [12]. CPP is a biomimetic saliva with a significantly greater calcium-stabilizing capacity than salivary proteins, due to higher content of its phosphoseryl residues. $\mathrm{CPP}$-amorphous calcium phosphate (CPP-ACP) nanocomplexes are readily soluble in saliva, acting as 
$\mathrm{Ca}^{2+}$ and $\mathrm{PO}_{4}{ }^{3-}$ reservoir, creating a diffusion gradient and a supersaturated level of ions in the oral cavity. Low $\mathrm{pH}$ conditions facilitate the release of $\mathrm{Ca}^{2+}$ and $\mathrm{PO}_{4}{ }^{3-}$ ions, inhibiting demineralization and contributing positively to the remineralization by precipitation of the released ions [5]. Published literature and systematic reviews on the CPP-ACP performances reached sometimes conflicting conclusions: some reviews suggest that $\mathrm{CPP}-\mathrm{ACP}$ had significant remineralizing and caries preventive effects; conversely, other systematic reviews conclude that the evidence to support its long-term remineralizing action or synergistic effect with fluoride is limited $[19,20]$. However, it has been suggested that the reason for the variability of the results obtained by using CPP-ACP, could be correlated to its remineralization mechanism, that involves an enhanced remineralization of enamel sub-surface lesions compared to predominantly surface-only remineralization produced by fluoride containing agents [21].

Given the different specific action of the two compounds and the absence of studies regarding their combined use, the present protocol for the evaluation of their potential synergic activity was designed. The treatment efficacy, in terms of remineralization potential and surface protection, was evaluated by means of profilometric and SEM analysis in an ex vivo model. Differences in surface resistance to acid attacks, before and after the treatment, were assessed by quantifying the release of $\mathrm{Ca}^{2+}$ using complexometric titration. The results obtained with the combined use of Elm-EP (Elm-EP, Colgate-Palmolive Co., New York, USA) and CG-TM (GC-TM, GC Co., Tokyo, Japan) were compared with those obtained using the sole Elm-EP or only artificial remineralizing saliva.

\section{Materials and Methods}

\subsection{Generality}

Table 1 shows the composition of the two considered remineralizing agents, Elm-EP and GC-TM, together with their suggested use as indicated by the manufacturer.

Table 1. Technical profile of the materials according to the manufacturer's data sheets.

\begin{tabular}{|c|c|c|}
\hline Material & Composition & Indication \\
\hline Elmex Erosion Protection (Elm-EP) & $\begin{array}{l}\text { Aqua, Hydrated Silica, Glycerin, Sorbitol, } \\
\text { Hydroxyethylcellulose, Aroma, } \\
\text { Cocamidopropyl Betaine, Olaflur, Sodium } \\
\text { Gluconate, Stannous Chloride, Alumina, } \\
\text { Chitosan, Sodium Saccharin, Sodium } \\
\text { Fluoride, Potassium Hydroxide, Hydrochloric } \\
\text { Acid, CI } 77891 \text {. Contains amine fluoride and } \\
\text { sodium fluoride (1400 ppm F-). }\end{array}$ & $\begin{array}{l}\text { Use toothpaste as usual according } \\
\text { to your daily oral hygiene habits: } \\
\text { brushing 2-3 times per day. }\end{array}$ \\
\hline GC Tooth Mousse (GC-TM) & $\begin{array}{c}\text { Pure water, Glycerol, } 10 \% \\
\text { casein-phospholipid and amorphous calcium } \\
\text { phosphate complex (CPP-ACP), D-sorbitol, } \\
\text { Silicon dioxide, Xylitol, Phosphoric acid, } \\
\text { Guar gum, Zinc oxide, Sodium saccharin, } \\
\text { Ethyl p-hydroxybenzoate, Magnesium oxide, } \\
\text { Butyl p-hydroxybenzoate, Propyl } \\
\text { p-hydroxybenzoate }\end{array}$ & $\begin{array}{l}\text { Apply to the teeth using a clean } \\
\text { finger or a cotton swab and let the } \\
\text { tooth work for two/three minutes, } \\
\text { then expector the excess and avoid } \\
\text { food or drink for } 30 \text { min. }\end{array}$ \\
\hline
\end{tabular}

During the experimental procedures, the wear condition of the specimen surfaces was evaluated quantitatively, by the profilometric analysis of the surface roughness ( $\mathrm{Sa}, \mu \mathrm{m})$, and qualitatively, by SEM analysis, at four different stages namely:

- $\mathrm{T} 0=$ baseline point/preparation of the specimens;

- $\quad \mathrm{T} 1$ = first erosive test;

- $\quad \mathrm{T} 2=15$ days of remineralization protocol;

- $\mathrm{T} 3=$ second erosive test. 
In order to preserve the original specimens during the experimental protocol, for each considered stage, epoxy resin clones (EpoThin Epoxy Resin+Hardener, Buehler, Lake Bluff, Illinois, USA) were prepared by using silicone molds (Type 3 impression material, AquasilUltra XLV; light-bodied consistency; Dentsply, York, Pennsylvania, USA) and used in the quantitative and qualitative analysis.

\subsection{Dental Specimens Preparation}

Twenty-four enamel specimens were obtained from 3-years-old cattle incisor teeth, by longitudinally sectioning the buccal-lingual fragments with a microtome (Micromet Remet, Bologna, Italy) equipped with a $0.5 \mathrm{~mm}$ thickness diamond blade. The buccal sections were discarded and from each lingual section, two parallelepipeds of an average size of $4 \times 2 \times 3 \mathrm{~mm}$ were obtained. The parallelepipeds were embedded in epoxy resin cylindrical supports, carefully leaving the dental surface exposed. The opposite surface of the resin support was fixed onto a glass slide (Figure 1). The surface of each specimen was polished for one minute with wet sand-paper of decreasing abrasive grain (\# 800, \# 1200, \# 2400). The surface conditions were verified using a 50x optical microscope (Leica MZ16, Leica Microsystems Srl, Milan, Italy), equipped with a digital camera (Leica DFC320, Leica Microsystems Srl, Milan, Italy), in order to eliminate specimens with defects and cracks. The obtained specimens were then randomly divided in three groups $(\mathrm{N}=8)$, which underwent different treatments (see following section) after the first erosive challenge at stage T1.

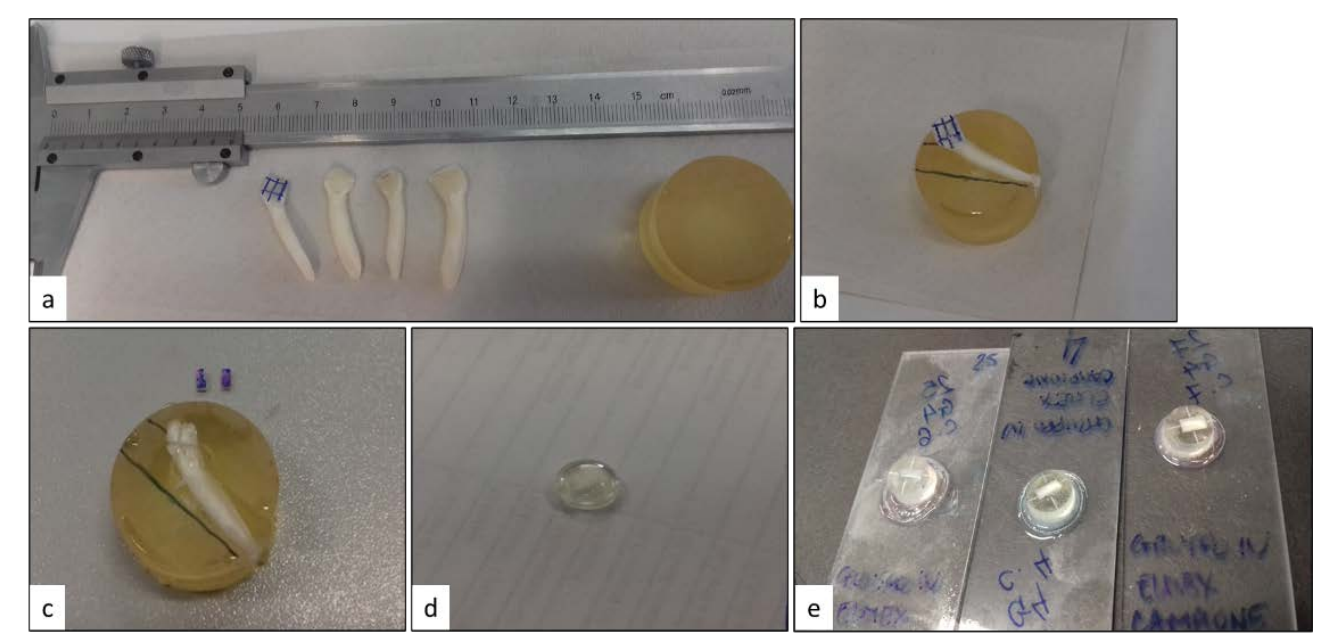

Figure 1. Specimens' preparation: $(\mathbf{a}, \mathbf{b})$ cattle incisor tooth supported by epoxy-resin cylinder for sectioning with a microtome; (c) 2 buccal-lingual fragment per tooth (in blue) obtained by the longitudinally sectioning with diamond blade; (d) specimen embedded in epoxy resin; (e) appearance of the specimens after polishing procedure.

\subsection{Erosive Challenge and $\mathrm{Ca}^{2+}$ Titration}

The specimens were subjected to the erosive challenge twice (T1 and T3) individually placing them into $\mathrm{HCl}$ aqueous solution $(0.01 \mathrm{M}, \mathrm{pH} 2,10 \mathrm{~mL})$ for $60 \mathrm{~min}$ at $37^{\circ} \mathrm{C}$.

For the titration of the loss $\mathrm{Ca}^{2+}, 3 \mathrm{~mL}$ aliquot of the $\mathrm{HCl}$ solution used for the erosion of the specimens was placed into a vial and 2-3 drops of $\mathrm{NH}_{3} / \mathrm{NH}_{4} \mathrm{Cl}$ buffer solution were added to $\mathrm{pH} 10$. Two drops of Eriochrome Black $\mathrm{T}$ was then added and immediately titrated against a standardized $0.001 \mathrm{M}$ EDTA solution to the red-blue indicator end-point, using a glass graduated burette $(50 \mathrm{~mL}$, precision $0.1 \pm 0.05 \mathrm{~mL}$, Hirschmann, Baden-Württemberg, Germany). Titration was repeated three times for each specimen. The concentration of free calcium ions in solution was reported as $\mathrm{mg} / \mathrm{L}$. 


\subsection{Treatment of the Specimens}

Specimen groups G0, G1 and G2 $(\mathrm{N}=8)$ underwent the following treatments for 15 days after the first erosion:

G0 specimens were stored in remineralizing saliva prepared as previously reported [22]. Briefly, $500 \mathrm{~mL}$ of artificial saliva contained: $\mathrm{Na}_{2} \mathrm{PO}_{4} 0.170 \mathrm{~g}$; sodium ascorbate $0.001 \mathrm{~g}$; glucose $0.015 \mathrm{~g}, \mathrm{NaCl}$ $0.290 \mathrm{~g}, \mathrm{CaCl}_{2} 0.085 \mathrm{~g}, \mathrm{NH}_{4} \mathrm{Cl} 0.080 \mathrm{~g}, \mathrm{KCl} 0.635 \mathrm{~g}, \mathrm{NaSCN} 0.080 \mathrm{~g}, \mathrm{KH}_{2} \mathrm{PO}_{4} 0.165 \mathrm{~g}$, urea $0.100 \mathrm{~g}$ and the final $\mathrm{pH}$ was adjusted to 7 .

G1 specimens were brushed twice a day (at 9 a.m. and at 8 p.m.) with Elm-EP using a soft toothbrush (Elmex Sensitive, Colgate-Palmolive Co., New York, USA) and following an adequate modified Bass technique performing a "rolling" number $(n=5)$ for each specimen $[23,24]$. Before rinsing with water, Elm-EP was left for two minutes onto the specimens surface.

G2 specimens were first treated with Elm-EP as described before for G1, then a rice size quantity of GC-TM was applied to each surface and left for $5 \mathrm{~min}$. The excess of paste was gently removed with a cotton swab.

G1 and G2 specimens were stored in artificial saliva when not retrieved for the treatments.

\subsection{Profilometric Assessment}

At each considered experimental stage (T0-T3) the resin clones were used for average surface roughness measurement $(\mathrm{Sa}, \mu \mathrm{m})$ by means of a profilometer (Talysurf CLI 1000; Taylor Hobson, AMETEK Inc, Berwyn, PA, USA) as previously described $[25,26]$. Approximatively in the middle area of each specimen a $0.5 \times 0.5 \mathrm{~mm}$ surface was analyzed (along horizontal lines), with a 501 point/line resolution and $1 \mu \mathrm{m}$ spacing; forward and return speeds were $200 \mu \mathrm{m} / \mathrm{s}$ and $500 \mu \mathrm{m} / \mathrm{s}$ respectively. The obtained data were processed with TalyMap Expert 4.1 profile software (Taylor Hobson Precision, Taylor Hobson, AMETEK Inc, Berwyn, PA, USA).

\subsection{Scanning Electron Microscope Assessment}

At any considered experimental stage (T0-T3), three resin clones per specimen group (G0-G2) were randomly chosen for surface qualitative evaluation by scanning electron microscopy. The clones were mounted on aluminum supports, coated with a carbon-conductive double-sided adhesive and then coated with a layer of gold with the metallizer (Sputter Coater K550X, Emitech, Quorum Technologies Ltd., Laughton, United Kingdom). The images were acquired with a scanning electron microscope (SEM, Quanta 250 SEM, FEI, Hillsboro, USA) operating in secondary electron acquisition mode. The working distance (WD) was modulated to obtain $5000 \times$ magnification; the acceleration voltage was set at $30 \mathrm{kV}$.

\subsection{Statistical Analysis}

Shapiro-Wilk test and Levene test were used for verifying the distribution normality and the variances homogeneity respectively. Statistically significant variations of the surface roughness parameter $(\mathrm{Sa})$ were evaluated among different groups at the same experimental stage and among the same group at different stages, by means of parametric ANOVA analysis of variance. Multiple comparisons were determined by post-hoc Tukey test, with pre-set significance level $p=0.05$. Statistically significant variations of the concentration of calcium $(\mathrm{mg} / \mathrm{L})$ in solution at $\mathrm{T} 1$ and $\mathrm{T} 3$ were evaluated by the non-parametric Kruskal-Wallis analysis. Pairwise comparison between dependent and independent groups of data was assessed by Wilcoxon and Mann-Whitney methods respectively, with pre-set significance level $p=0.05$. 


\section{Results}

\subsection{Release of $\mathrm{Ca}^{2+}$}

The box-plot graphic in Figure 2, shows the concentration of $\mathrm{Ca}^{2+}$ in $\mathrm{mg} / \mathrm{L}$, obtained by the EDTA titration method. As expected, despite the use of the same protocol for the preparation of the specimens, the concentration of $\mathrm{Ca}^{2+}$ titrated after the first acid challenge was statistically different among groups $(p<0.05)$. This result can be ascribable to small differences in the specimens size and to the intrinsic variability of the biologic dental matrix. For this reason, only significative variations $(p<0.05)$ of the concentration of $\mathrm{Ca}^{2+}$ at T1 and T3 in the same group will be considered and discussed. In case of G0 specimens the concentration of $\mathrm{Ca}^{2+}$ at $\mathrm{T} 1$ was only slightly higher than that at $\mathrm{T} 3$, but statistically different $(p=0.046)$. For both G1 and G2 specimens the concentration of $\mathrm{Ca}^{2+}$ lost at T3 was significantly lower compared to that titrated at T1 $(p<0.05)$, decreasing of $44 \%$ and $22 \%$ respectively.

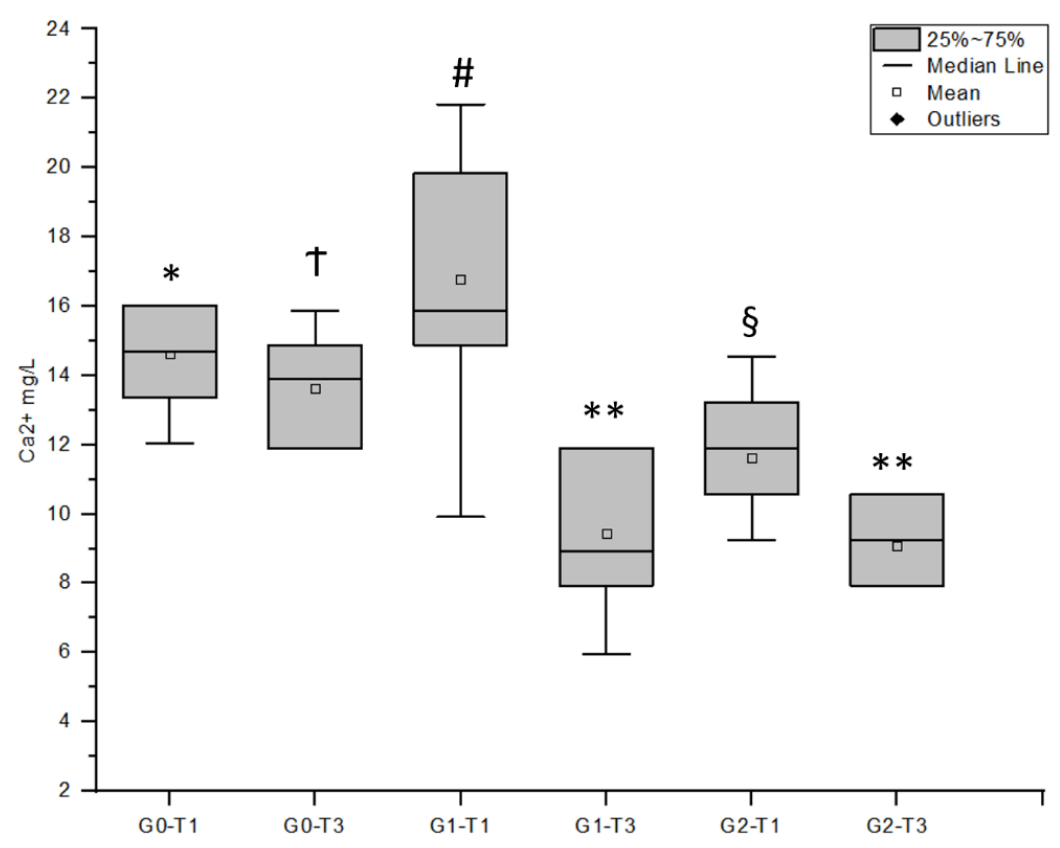

Figure 2. Concentration of $\mathrm{Ca}^{2+}$ in $\mathrm{mg} / \mathrm{L}$ released by the different groups during the two erosive challenges (T1 and T3), evaluated by the EDTA titration method. Same symbol above the box-plot indicates no statistical difference between data $(p>0.05)$.

\subsection{Quantitative Assessment}

Table 2 shows the values of the average surface roughness (Sa) of the considered groups at the various experimental stages obtained by means of profilometry.

At T0 and T1 the Sa parameter was no significantly different among specimens of different groups, $(p>0.05)$. Sa value at $\mathrm{T} 2$ proved to be treatment-dependent: $\mathrm{Sa} \mathrm{G}_{\mathrm{G} 0}$ was significantly higher than Sa $\mathrm{G} 1$ and $\mathrm{Sa}_{\mathrm{G} 2}(p<0.05)$ which, instead, were no significantly different from each other $(p>0.05)$. The Sa parameter showed the same statistical trend at $\mathrm{T} 3: \mathrm{Sa}_{\mathrm{G} 0}>\mathrm{Sa}_{\mathrm{G} 1}=\mathrm{Sa}_{\mathrm{G} 2}$.

Considering the experimental stages T0-T3, all the groups showed a significant difference $(p<0.05)$ between the Sa evaluated at T0 and T1: irrespectively from the considered group $\mathrm{Sa}_{\mathrm{T} 1}>\mathrm{Sa}_{\mathrm{T} 0}(p<0.05)$.

In general, the variation of $\mathrm{Sa}$ among the different experimental stages was treatment-related: the statistical trend of Sa value for $\mathrm{G} 0$ can be resumed as $\mathrm{Sa}_{\mathrm{T} 0}<\mathrm{Sa}_{\mathrm{T} 1}=\mathrm{Sa}_{\mathrm{T} 2}<\mathrm{Sa}_{\mathrm{T} 3}$. In case of G1: Sa $\mathrm{T}_{2}$ was statistically lower than $\mathrm{Sa}_{\mathrm{T} 1}(p<0.05)$, while $\mathrm{Sa}_{\mathrm{T} 3}$ was not significantly different $(p>0.05)$ from both $\mathrm{Sa}_{\mathrm{T} 1}$ and $\mathrm{Sa}_{\mathrm{T} 2}$. G2 specimens showed a significant decrease of $\mathrm{Sa}_{\mathrm{T} 2}$ in comparison to Sa $\mathrm{T}_{\mathrm{T} 1}(p<0.05)$ and, after the second erosion, a subsequent increase, leading to no statistical difference between the final $\mathrm{Sa}_{\mathrm{T} 3}$ and $\mathrm{Sa}_{\mathrm{T} 1}(p>0.05)$. 
Table 2. Surface roughness parameter obtained by means of profilometry at the different stages of the experimental protocol.

\begin{tabular}{ccccc}
\hline & \multicolumn{4}{c}{ Sa $[\mu \mathrm{m}]^{*}$} \\
\cline { 2 - 5 } & T0 & T1 & T2 & T3 \\
\hline G0 & $0.129 \pm 0.018^{\mathrm{Aa}}$ & $0.502 \pm 0.085^{\mathrm{Ab}}$ & $0.494 \pm 0.098^{\mathrm{Ab}}$ & $0.748 \pm 0.101^{\mathrm{Ac}}$ \\
\hline G1 & $0.127 \pm 0.022^{\mathrm{Aa}}$ & $0.498 \pm 0.126^{\mathrm{Ab}}$ & $0.286 \pm 0.040^{\mathrm{Bc}}$ & $0.390 \pm 0.092^{\mathrm{Bbc}}$ \\
\hline G2 & $0.142 \pm 0.006^{\mathrm{Aa}}$ & $0.520 \pm 0.098^{\mathrm{Ab}}$ & $0.258 \pm 0.039^{\mathrm{Bc}}$ & $0.455 \pm 0.110^{\mathrm{Bb}}$ \\
\hline
\end{tabular}

$\mu \mathrm{m}$, square micrometer; $\mathrm{Sa}$, roughness parameter values of area. ${ }^{*}=\mathrm{Sa}$ are expressed as mean values \pm standard deviations; $\mathrm{N}=8$ specimens per group. Means followed by the same superscript capital letter indicate no statistical differences in column $(p>0.05)$; means followed by the same superscript lowercase letter indicate no statistical differences in row $(p>0.05)$.

\subsection{Qualitative Assessment}

Figure 3 shows the SEM morphologic changes characterizing the specimens of the different groups during different experimental stages. SEM images are representative of the specimen portion closest to the cervical region of the tooth. Irrespectively from the considered group, surfaces appeared smooth at T0, polished and homogeneous. The acid challenge at T1 caused a deep erosion of the enamel surface partially exposing dentin tubules in all the considered groups. Among groups, a different surface morphology appeared at T2, correlated to the used treatment: G0 specimens showed a slight surface remineralization, not sufficient to mask the dentin tubules; G1 specimen surfaces were more compact and homogeneous, similar to the baseline at $\mathrm{T} 0$ of the same group, with the dentin tubules efficiently occluded; G2 specimens showed a well remineralized surface, with complete occlusion of the dentin tubules, CPP-ACP cluster can be recognized onto the surface. At T3, the G0 specimen surfaces were drastically eroded, with dentin tubules exposure and visible roughness increase; G1 specimen surfaces showed to resist the additional erosion thanks to an effective protective layer which prevented dentin tubules exposure; in the G2 specimens the CPP-ACP clusters previously visible onto the surfaces were mostly removed by the acid solution, although the sub-surface layer resisted, protecting the underlying dentin tubules from exposure.
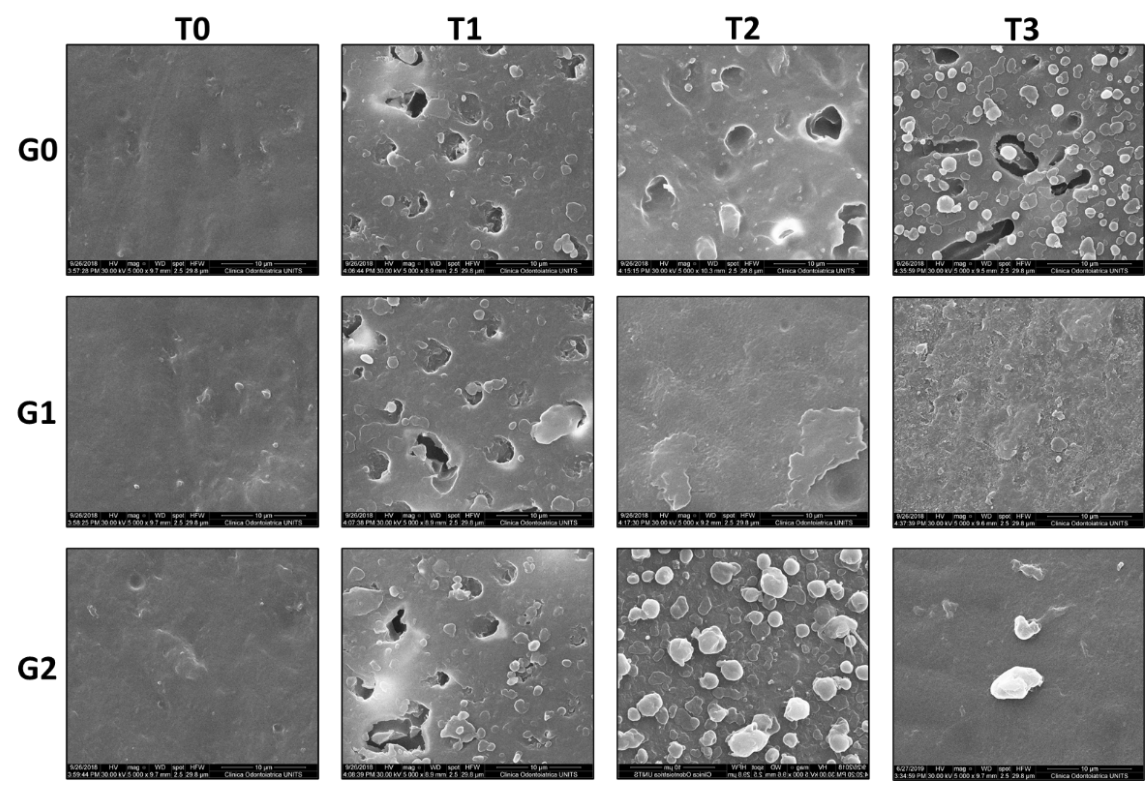

Figure 3. SEM assessment of the changes in surface appearance of the three groups (G0, G1, G2), during the different experimental stages (T0, T1, T2, T3). Scale bar $10 \mu \mathrm{m}$. 


\section{Discussion}

In the present work, a possible advantage correlated to the synergic use of Elm-EP and GC-TM, two commonly used home-devices acting in different mode on eroded tooth surface, was investigated.

In order to mimic conditions of patients afflicted by diseases such as GERD and BN, the specimens were retrieved from frontal dental elements, as they represent the most affected elements by erosion. Furthermore, the acid experimental test was extended to $60 \mathrm{~min}$ to reach partially exposed dentine tubules, reproducing the situation of critically eroded tooth surfaces. The specimens were stored in artificial saliva both after preparation and during the entire treatment period reproducing the natural environment of the mouth. As negative reference group (G0), the specimens where stored in remineralizing saliva for 15 days, while as positive reference (G1) the specimens were treated with the sole toothpaste Elm-EP, which efficacy as anti-erosion agent has been largely assessed and reported in literature $[8,9,11]$. The two products were applied on the specimen surfaces following the methodologies suggested by the manufacturer.

The results obtained in this study highlight that the combined use of Elm-EP and GC-TM for 15 days on critically eroded specimens, allow to greatly decrease the surface roughness. Even though, the Sa values of the specimens treated with Elm-EP or its combination with GC-TM were not statistically different, the calculated percentage of decrease in roughness at $\mathrm{T} 2$ compared to $\mathrm{T} 1$, resulted $42 \%$ and $50 \%$ for G1 and G2 respectively, confirming the promising action of the combined agents. Together with the morphologic analysis obtained by SEM evaluation, the profilometric analysis allows to speculate about the formation of a protective layer obtained by the Elm-EP active components in which some $\mathrm{CPP}-\mathrm{ACP}$ clusters inserted, contributing to the increase of the surface uniformity.

Considering the severe erosion conditions applied to the specimens, not surprisingly, 15 days of treatment with the studied agents proved to be insufficient to restore the baseline Sa value, but as the action of both Elm-EP and GC-TM is time-dependent, it is possible to hypothesize a gradual decrease of the roughness by increasing the treatment period.

The evaluation and comparison of the released calcium concentration during the two acid attacks gave some information about the erosion-protecting attitude of the considered agents: the solubilized calcium titrated at T3, in case of the positive reference group G1, was greatly lower (44\%) compared to that at T1, confirming the well-known ability of Elm-EP as anti-erosive system [9]. However, G1 specimen surfaces appeared less homogeneous at T3 in comparison to the baseline and T2. Differently, in case of G2, the concentration of $\mathrm{Ca}^{2+}$ lost during the second erosion was only $22 \%$, lower than that titrated at T1. It is possible to speculate about this result comparing the SEM imagines regarding G2 at T2 and T3: after the second erosion, the most part of the CPP-ACP clusters, well visible at T2, disappeared from the specimen surfaces, suggesting their dissolution in the acid media. Bearing in mind that the CPP-ACP nanocomplexes are meaning to act as $\mathrm{Ca}^{2+}$ and $\mathrm{PO}_{4}{ }^{3-}$ storage-release systems, it is plausible to consider that the most percentage of the calcium released and titrated at T3 originated from their dissolution instead of that of the tooth surface. Nevertheless, G2 specimens showed still occluded dentine tubule after the second drastic acid erosion, confirming, ones more, the remineralization process of the two combining agents.

The phenomenon of CPP-ACP dissolution, could also be correlated to the different percentage of roughness increase of G1 (26\%) and G2 (76\%) at T3: assuming that, thanks to the different remineralizing action of Elm-EP and GC-TM, a "mixed layer" nestling CPP-ACP clusters could form onto G2 specimen, in case of an acid attack, not only the CPP-ACP clusters on the surface would be removed, but also the ones included in the "mixed layer", increasing the surface roughness.

Even though the tandem use of Elm-EP and GC-TM gave indeed promising results, the present study evidenced a poor stabilization of the CPP-ACP clusters onto the specimen surface in the considered conditions. To justify this trend, chemical and mechanical interaction between the active components of Elm-EP and GC-TM should be deeper studied. However, it is possible to speculate about some explanations: for instance, it has been previously reported that for obtaining optimal remineralization and protection results, CPP should bind to salivary pellicle and plaque bacteria [27]. 
It is then possible, that the formation of a thin film of Elm-EP on the specimen surfaces would have limited the bonding process, decreasing the cluster stability to the acid attack. Moreover, the inclusion of the CPP-ACP nanocomplexes in the formation of the assumed "mixed layer" could be insufficient for their stability in comparison to the presence of the biologically complex matrix typical of an in vivo environment.

The complete dissolution of CPP-ACP nanocomplexes and the consequent limitation of the GC-TM protection efficiency, seem to be in disagreement with some results reported in literature, where the use of CPP-ACP-containing systems showed a clear effect against enamel demineralization in vitro [28,29]. However, it is important to keep in mind that data discrepancies among studies can be caused by the use of different experimental protocols, for instance, the use of bovine teeth model instead of human specimens. Even though bovine and human enamels possess many similarities, such as chemical compositions and physicochemical properties, the different arrangements of prisms and interprisms might result in some different outcomes [30]. Moreover, bovine enamel is more porous than human enamel, probably because it consists of larger crystals [31], and these morphological aspects may be correlated to the demineralization of bovine enamel which progresses about three times faster than that of human enamel. Therefore, the performance of the materials in human enamel may be different from the results of our study.

\section{Conclusions}

Within the limitations of the present in vitro study, the combined use of the two home-devices, Elm-EP and CPP-ACP, in the treatment of severe eroded teeth showed to be promising. In particular, for what the surface roughness recovery concerns, the simultaneously use of the two agents gave superior results in comparison with the use of the sole Elm-EP, decreasing the surface roughness of $50 \%$ in 15 days and implying an efficient remineralization process. The effectiveness in tooth protection from additional surface erosion should be deeper investigated with the aim of better understanding the interaction between the two different systems and possibly increasing their performance.

Author Contributions: Conceptualization, L.F. and F.C.; Data curation, G.M.; Formal analysis, L.F. and F.B.; Investigation, L.F. and L.P.; Methodology, G.M.; Project administration, V.N.; Supervision, F.C., R.D.L. and V.N.; Validation, R.D.L.; Visualization, F.B.; Writing—original draft, F.C.; Writing—review \& editing, F.C., F.B., G.M. and V.N. All authors have read and agreed to the published version of the manuscript.

Funding: The Authors declare to have not received any fund for this study.

Conflicts of Interest: The authors declare no conflict of interest.

\section{References}

1. Featherstone, J.; Lussi, A. Understanding the Chemistry of Dental Erosion. Monogr. Oral Sci. 2006, 20, 66-76. [PubMed]

2. Neel, E.A.; Aljabo, A.; Strange, A.; Ibrahim, S.; Coathup, M.; Young, A.; Bozec, L.; Mudera, V. Demineralization-remineralization dynamics in teeth and bone. Int. J. Nanomed. 2016, 11, 4743-4763. [CrossRef] [PubMed]

3. Kanzow, P.; Wegehaupt, F.; Attin, T.; Wiegand, A. Etiology and pathogenesis of dental erosion. Quintessence Int. 2016, 47, 275-278. [PubMed]

4. Rakhmatullina, E.; Bossen, A.; Höschele, C.; Wang, X.; Beyeler, B.; Meier, C.; Lussi, A. Application of the specular and diffuse reflection analysis for in vitro diagnostics of dental erosion: Correlation with enamel softening, roughness, and calcium release. J. Biomed. Opt. 2011, 16, 107002. [CrossRef] [PubMed]

5. Nebu, P. State of the Art Enamel Remineralization Systems: The Next Frontierin Caries Management. Caries Res. 2019, 53, 284-295.

6. Neveena, P.; Nagarathana, C.; Sakunthala, B. Remineralizing Agent-Then and Now-An Update. Dentistry 2014, 4, 5 . 
7. Mittal, R.; Relhan, N.; Tangri, T. Remineralizing Agents: A Comprehensive Review. Int. J. Clin. Prev. Dent. 2017, 13, 1-4. [CrossRef]

8. Ganss, C.; von Hinckeldey, J.; Tolle, A.; Schulze, K.; Klimek, J.; Schlueter, N. Efficacy of stannous ion and byopolymer in toothpases on enamel erosion/abrasion. J. Dent. 2012, 40, 1036-1043. [CrossRef]

9. Aykut-Yetkiner, A.; Attin, T.; Wiegand, A. Prevention of dentine erosion by brushing with anti-erosive toothpastes. J. Dent. 2014, 42, 856-861. [CrossRef]

10. Yonel, N.; Bikker, F.; Lagerweij, M.; Kleverlaan, C.; van Loveren, C.; Ozen, B.; Çetiner, S.; van Strijp, A.J. Anti-erosive effects of fluoride and phytosphingosine: An in vitro study. Eur. J. Oral Sci. 2016, 124, $396-402$. [CrossRef]

11. Wasser, G.; Joao-Souza, S.; Lussi, A.; Carvalho, T. Erosion-protecting effect of oral-care products available on the Swiss market. Swiss Dent. J. SSO 2018, 128, 290-296.

12. Sanavia, C.; Tatullo, M.; Bassignani, J.; Cotellessa, S.; Fantozzi, G.; Acito, G.; Iommiello, A.; Chiavistelli, L.; Sabatini, S. Nardi GM Remineralization Strategies in Oral Hygiene: A Position Paper of Italian Society of Oral Hygiene Sciences-S.I.S.I.O. Working Group. Open Dent. J. 2017, 11, 527-538. [CrossRef] [PubMed]

13. Bae, K.; Jun, E.; Lee, S.; Paik, D.; Kim, J. Effect of water-soluble reduced chitosan on Streptococcus mutans, plaque regrowth and biofilm vitality. Clin. Oral Investig. 2006, 10, 102-107. [CrossRef] [PubMed]

14. Verkaik, M.; Busscher, H.; Jager, D.; Slomp, A.; Abbas, F.; van der Mei, H. Efficacy of natural antimicrobials in toothpaste formulations against oral biofilms in vitro. J. Dent. 2011, 39, 218-224. [CrossRef]

15. Lee, H.; Tsai, S.; Kuo, C.; Bassani, A.; Pepe-Mooney, B.; Miksa, D.; Masters, J.; Sullivan, R.; Composto, R.J. Chitosan adsorption on hydroxyapatite and its role in preventing acid erosion. J. Colloid Interface Sci. 2012, 385, 235-243. [CrossRef]

16. Arnaud, T.; Barros-Neto, B.; Diniz, F. Chitosan effect on dental enamel de-remineralization: An in vitro evaluation. J. Dent. 2010, 38, 848-852. [CrossRef]

17. Keegan, G.; Smart, J.; Ingram, M.; Barnes, L.; Burnett, G.; Rees, G. Chitosan microparticles for the controlled delivery of fluoride. J. Dent. 2012, 40, 229-240. [CrossRef]

18. Ganss, C.; Schlueter, N.; Hardt, M.; Schattenberg, P.; Klimek, J. Effect of Fluoride Compounds on Enamel Erosion in vitro: A Comparison of Amine, Sodium and Stannous Fluoride. Caries Res. 2008, 42, 2-7. [CrossRef]

19. Wang, Y.; Li, J.; Sun, W.; Li, H.; Cannon, R.; Mei, L. Effect of non-fluoride agents on the prevention of dental caries in primary dentition: A systematic review. PLOS ONE 2017, 12, e0182221. [CrossRef]

20. Fontana, M. Enhancing fluoride: Clinical human studies of alternatives or boosters for caries management. Caries Res. 2016, 50 (Suppl. 1), 22-37. [CrossRef]

21. Shen, P.; Manton, D.; Cochrane, N.; Walker, G.; Yuan, Y.; Reynolds, C.; Reynolds, E.C. Effect of added calcium phosphate on enamel remineralization by fluoride in a randomized controlled in situ trial. J. Dent. 2011, 39, 518-525. [CrossRef] [PubMed]

22. Hannig, C.; Hamkens, A.; Becker, K.; Attin, R.; Attin, T. Erosive effects of different acids on bovine enamel: Release of calcium and phosphate in vitro. Arch Oral Biol. 2005, 50, 541-552. [CrossRef] [PubMed]

23. Bass, C. An effective method of personal oral hygiene. J. Louis Med. Soc. 1954, 106, 100-112.

24. Poyato-Ferrera, M.; Segura-Egea, J.; Bullón-Fernández, P. Comparison of modified Bass technique with normal toothbrushing practices for efficacy in supragingival plaque removal. Int. J. Dent. Hyg. 2003, 1, 110-114. [CrossRef]

25. Lohbauer, U.; Reich, S. Antagonist wear of monolithic zirconia crown after 2 years. Clin. Oral Investig. 2017, 4, 1165-1172. [CrossRef]

26. Baroni, C.; Marchionni, S.; Bazzocchi, M.G.; Cadenaro, M.; Nucci, C.; Manton, D.J. A Sem and non-contact surface white light profilometry in vivo study of the effect of a creme containing CCP-ACP and fluoride on young enamel. Scanning 2014, 36, 270-277.

27. Reynolds, C.; Cai, F.; Shen, P.; Walker, G. Retention in plaque and remineralization of enamel lesions by various form of calcium in mounthrinse or sugar free chewing gum. J. Dent. Res. 2003, 82, 206-211. [CrossRef]

28. Poggio, C.; Lombardini, M.; Dagna, A.; Chiesa, M.; Bianchi, S. Protective effect on enamel demineralization of CPP-ACP paste: An AFM in vitro study. J. Dent. 2009, 37, 949-954. [CrossRef]

29. Ranjitkar, S.; Kaidonis, J.; Richards, J.; Townsend, G. The effect of CPP-ACP on enamel wear under severe erosive conditions. Arch Oral Biol. 2009, 54, 527-532. [CrossRef] 
30. Wang, C.; Li, Y.; Wang, X.; Zhang, L.; Tiantang, F.B. The Enamel Microstructures of Bovine Mandibular Incisors. Anat. Rec. 2012, 295, 1698-1706. [CrossRef]

31. Arends, J.; Jongebloed, W.L. Crystallites dimensions of enamel. J. Biol. Buccale 1978, 6, 161-171. [PubMed] 